\title{
Genomic Epidemiology of BRCA1/BRCA2; Breast Cancer Associated Genes and Use of Electronic Health Record to Reduce the Escalating Cost of Treatment
}

\author{
E. William Ebomoyi ${ }^{1^{*}}$ \\ ${ }^{1}$ Department of Health Studies, College of Health Sciences, Chicago State University, \\ Chicago, Illinois 60628-1598, United States of America.
}

Review Article

Received $23^{\text {rd }}$ July 2011

Accepted 26 $6^{\text {th }}$ July 2011

Online Ready $31^{\text {st }}$ July 2011

\section{ABSTRACT}

This project reviewed gene mutations which are associated with BRCA1 and BRCA2. From epidemiological perspective, the escalating cost of breast cancer management is due to the aging phenomenon of the female cohort in United States population. Innovative genomic sequencing techniques could continue to augment gene mutations beside the BRCA1/BRCA2 and TP53, PTEN, ST11/LKB1, CDH1, CHEK2, and ATM among others. To reduce the escalating cost of breast cancer management, the application of pharmacogenomic techniques, use of electronic health record and the most appropriate software could be applied for both primary prevention and chemoprevention. Physicians and other clinicians must hone their skills in advanced Mendelian genetics to become proficient in using statistical risk analysis and the appropriate IT software to recommend primary prevention to at risk breast cancer patients.

Keywords: Genomic epidemiology; breast cancer; BRCA1; BRCA2; 


\section{INTRODUCTION}

Genomic epidemiology is defined as the investigation of the actions of genetics factors in determining health and disease onset in families and population and the interplay of genetics and environmental characteristics of people. Genomic epidemiology is the link uniting the intersection between genetic and molecular epidemiology. It focuses on the determinants and distribution of diseases and injuries in human population (Ebomoyi, 2009; Mausner and Bahn, 1987).

\section{EPIDEMIOLOGY OF BRCA1 AND BRCA2}

Although the normal function of breast cancer susceptibility gene1 and breast cancer susceptibility gene2 (BRCA1 and BRCA2) is to assist in repairing damaged DNA and inheritance for both genes is autosomal dominant with higher penetrance in women (Mikail, 2008). The American Cancer Society gave an estimate of 562,340 cancer deaths in US, made up of 292,540 males and 269,800 females (American Cancer Society, 2009). Cancer sites in men for which African-American recorded deaths $(313.0 / 100,000)$ by far exceed those of their Caucasian counterpart with rates of $230.7 / 100,000$. In the U.S., the financial expenditure on cancer alone, by the government is over $\$ 104$, billion dollars annually. Statistics from the National Cancer Institute gave an estimate of new cases of cancer each year to be 1,221,800 (National Cancer Institute, 2002). Based on the American Cancer Society, over $90 \%$ of all cancers are not necessarily genetic in nature. This group of cancers includes breast, lung, stomach, colon, skin and prostate cancers (American Cancer Society, 2009). The associated etiological agents for breast cancer are age, use of excessive amount of alcohol, exposure to radiation, chemotherapy, hormone therapy, immunotherapy, radionucleotides, and use of birth control pills, physical inactivity, obesity, and ingestion of dietary lipids. Warning signs of the cancers are sores that do not heal, unusual bleeding, lump in the breast, changes in the size of warts, and chronic cough.

Genomics interventions and the application of magnetic resonance imaging (MRI) have revealed the genetic association between BRCA1 and the BRCA2 and the onset of ovarian cancer. There are various malignant growths that begin in the breast with ductal-carcinoma and lobular carcinoma portraying; stage 1 , stage 2 and stage 3 which are most receptive to primary, secondary prevention and prophylactic treatment if detected early. From epidemiological studies, gender is the single most important risk factor for breast cancer, but that does not rule out the onset of breast cancers in males. Family history and genetic etiology are common with BRCA1 and BRCA2. Hormonal replacement therapy, induced radiation therapy, obesity contribute to the onset of breast cancer. However, the crucial and most effective intervention against cancer is early detection through breast self examination, family history technique, clinical assessment and mammograms performed at least twice a year by a competent oncologist.

By year 2030 or sooner, with the use of global genomic tools, geneticists will have the capability to precisely identify hundreds of genes with variations that predispose several clients to a broad spectrum of diseases such as cancerous, immunological, mental and metabolic diseases. Based on the scientific understanding of molecular biology, certain mutations have very high probability of causing specific diseases; while others merely predispose individuals to the onset of some diseases. In Sharp contrast, a mutation in breast cancer BRCA1, gene has only $70 \%$ chance of causing breast cancer in a 60 -year-old woman carrying one copy of the mutant gene. This disease state usually occurs if and only if 
the BRCA1 gene interacts with sundry environmental factors that affect the probability of activating the cancerous conditions. The other alternative is, it may occur if other genes modify expression of the BRCA1 gene. Medical geneticists and other clinicians can use this knowledge about the multiple factors contributing to the predisposition to diseases as relevant indicators in designing appropriate therapy for the individual based on the subject's DNA profile before the onset of breast cancer. The other salient example involves families of hereditary colon cancer detected through early screening. The odds of dying from such cancer can be modified through nutritional intervention and counseling. This era of predictive medicine could necessitate the use of therapeutic drugs to block or reverse the lethal effects of mutant genes (USPSTF, 2005). The United States Preventive Service Task Force (USPSTF) recommends that women whose family history is associated with high risk of lethal mutations in BRCA1or BRCA2 genes be referred for genetic counseling and evaluation for BRCA testing (United States Department of Health and Human Services, 2011).

With the use of cutting edge DNA analysis, it is possible for genetic epidemiologists, clinicians and public health scientists to establish the probability of an individual developing a disease based on an established intra-familial pattern. For example, through the assessment of family history of breast cancer patients, screening of individuals under 50 years of age and participation in comprehensive genetic evaluation, it is possible to avoid the lethal consequences of this type of cancer. Use of predictive medical sciences can protect many clients from various types of cancers. In 2008, The National Institutes of Health indicated how cancer disease burden cost the United States nearly $\$ 210$ billion; which included $\$ 74$ billion in direct medical costs, $\$ 17.5$ billion for lost productivity from illness, and $\$ 118.4$ billion for productivity loss due to unnecessary and premature death (United States Department of Health and Human Services, 2011). Colossal sums of money could be saved by adopting primary preventive measures. Over $\$ 104$ billion dollars can be saved annually from the nation health care budget by encouraging the public through health education to participate in self examination, and create a calendar for preventive health screening that is appropriate for one's age, gender, and cultural precept through the consultation of one's health care provider.

Particularly, out-patient care is a tremendous cost saving measure because of the inflated cost of hospital beds, exposure to unnecessary superfluous medical technologies and the inadvertent risk of nosocomial and iatrogenic diseases which increase national medical expenses exponentially. Through self-examination and health education, one is able to avoid the emergency room services for life-threatening diseases. Besides, such emergency services are two to three times more costly than services provided in the comfort of a visit to one's family physician. Genetic testing exists for the identification of mutation in affected patients and their families. Recent genome-based investigations have yielded relevant information about population specific mutation in BRCA1 genes that occur with high frequencies in different ethnic groups and their haplotype characteristics Malone et al. (2011) in their epidemiological study found that the prevalence and predictors of BRCA1/BRCA2 mutations were more common in White women compared to American women of African ancestry. Statistically, BRCA1 mutations were significantly more common in White $(2.9 \%)$ compared to (1.4\%) in Black women. The deleterious genes in Jewish cases were $10.2 \%$ compared to the non-Jewish subjects. However, BRCA2 mutations were slightly more frequent in African-American women compared to Caucasian (2.1\%) female subjects. 
In view of the lethal impact of breast cancer genes, the study described here was designed to:

- Explore the genomic characteristics of BRCA1 and BRCA2 mutations

- Identify the modifiable and non-modifiable genetic risks associated with breast cancer

- Pinpoint the contributory factors escalating the cost of breast cancer management and

- Identify succinct techniques for utilizing electronic health record for reducing the exorbitant cost of medical treatment of breast cancer.

\section{GENOMICS SEQUENCING OF BRCA1/BRCA2}

In genomic medicine, BRCA1 and BRCA2 are not the only genetic mutations that increase the risk of breast cancer. Innovative sequencing recently revealed many other genes such as the TP53, PTEN, STK11/LKB1, CDH1, CHEK2, ATM, MLH1 and MSH2. The genomewide association studies of breast cancer recently identified multiple single gene nucleotide polymorphisms (SNPs) that are associated with increased incidence of breast cancer in the general population. Some of the alleles at the three of these SNPs in FGFR2, TNRCq, and MAP3K increased risk of breast cancer for BRCA1 and BRCA2 mutation carriers. In the general population, beside those reported with higher prevalence rates, three specific SNPs rs3817198 at LSP1 and rs13387042 at 8q24 have also been shown to associate with onset of breast cancer (Malone et al, 2011; Antoniou et al., 2009).

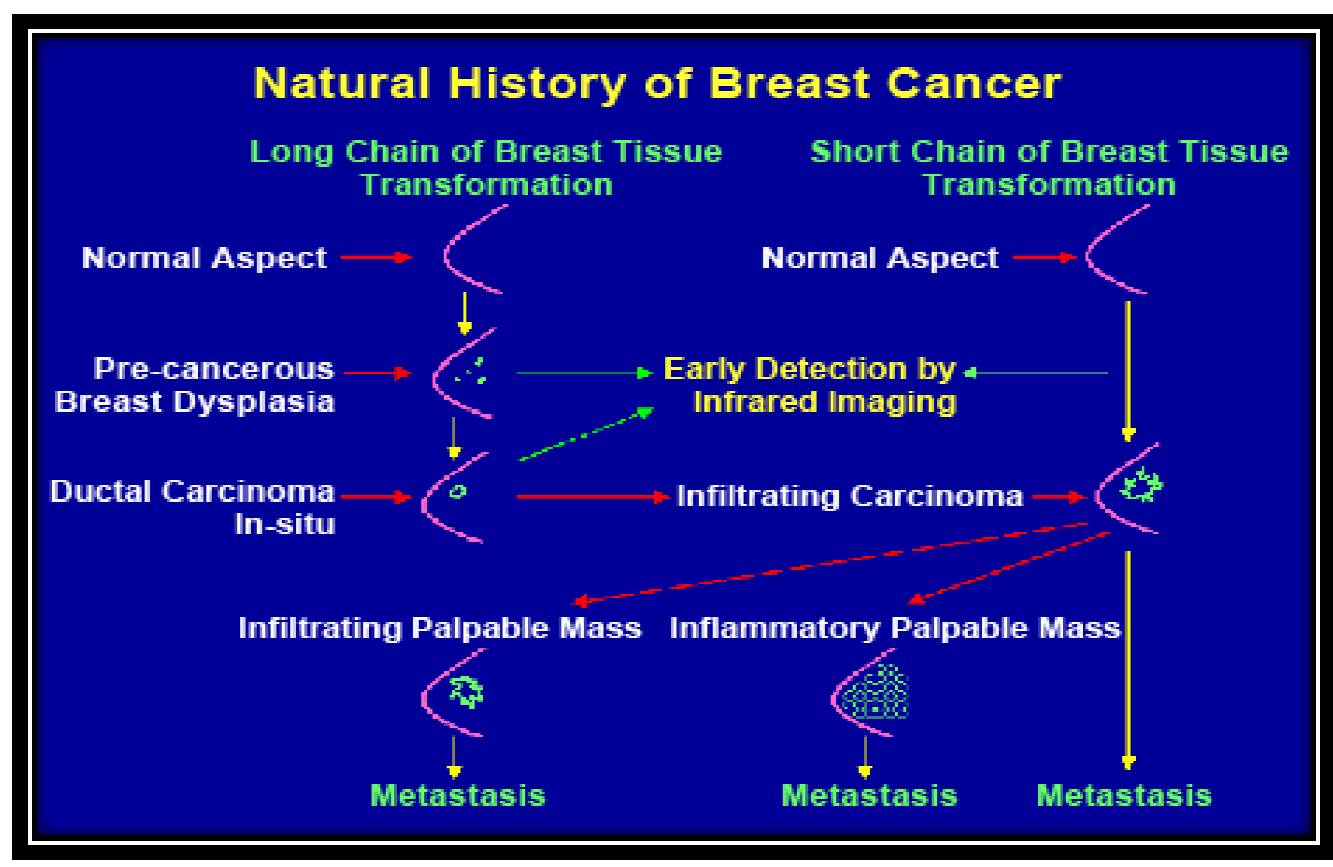

Fig. 1. Natural History of Breast Cancer 
These recently detected mutations account for only a fraction of hereditary breast cancer. These gene-mutations account for at least 5 to $10 \%$ of the total ovarian cancers among Caucasian women in United States. The BRCA2 is located on chromosome 13 and the risk of developing breast cancer in women diagnosed with BRCA2 mutations is similar to the risk of BRCA1 patients (Antoniou et al., 2009). The genome-wide association (GWA) studies of breast cancer continue to identify multiple single nucleotide polymorphisms, which are associated with increased risk in the general population. A detailed account of such global genomic study is beyond the scope of this project.

\section{MORTALITY STATISTICS}

The American Cancer Society gave an estimate of 562,340 cancer deaths in US, made up of 292,540 males and 269,800 females (American Cancer Society, 2009). Cancer sites in men for which African-American recorded deaths $(313.0 / 100,000)$ by far exceed those of their Caucasian counterpart with rates of $230.7 / 100,000$. In the U.S., the money expended on cancer alone, by the government is over $\$ 104$, billion dollars annually. The National Cancer Institute gave an estimate of new cases of cancer each year to be 1,221,800 (Mariotto, 2011).

As revealed by the American Cancer Society, over $90 \%$ of all cancers are not necessarily genetic in nature (American Cancer Society, 2009). This group of cancers includes breast, lung, stomach, colon, skin and prostate cancers. The etiological agents of these cancers are largely due to tobacco and environmentally induced. Other etiological agents are age, use of excessive amount of alcohol, exposure to radiation and radio-nucleotides, chemotherapy, hormone therapy, immunotherapy, radio-nucleotides. The warning signs of the cancers are sores that do not heal, consistent history of breast cancer in the family, unusual bleeding, lump in the breast, changes in the size of warts, and chronic cough.

Genomics interventions and the application of MRI have revealed the genetic association between BRCA1 and the BRCA2 and the onset of ovarian cancer. There are various malignant growths that begin in the breast with ductile carcinoma and lobular carcinoma portraying; stage 1, stage 2 and stage 3 which are most receptive to primary, secondary prevention and prophylactic treatment if detected early (Figure 1). From epidemiological studies, gender is the single most important risk factor for breast cancer, but that does not rule out the onset of breast cancers in males. Family history and genetic etiology are common with BRCA1 and BRCA2. Hormonal replacement therapy, iatrogenic induced radiation therapy, obesity are also causes of cancer. However, the crucial and most effective intervention against cancer is early detection through breast self examination, clinical assessment and mammograms performed at least twice a year. The non-modifiable risk factors are the inherited genes, intra-familial characteristics, parity, age, exposure to radionucleotide, reproductive history, menstrual history and ethnicity. The modifiable risk factors include obesity, on-going exercise, breastfeeding, use of alcohol, hormone replacement therapy, use of birth control pills and infertility or parity status.

\section{COPING WITH TRENDS IN THE ESCALATING COST OF BREAST CANCER}

Angela Mariotto reported that the cost of management of cancer could exceed $\$ 200$ billion by 2020 (Mariotto et al., 2011). Although the incidence and survival from cancer have improved in recent years, more expensive technology and the changing population characteristics have contributed to the increasing cost of cancer treatment. With the elderly 
population constantly growing, the cost of cancer management will continue to increase. By 2011, The National Institutes of Health has projected the cancer costs could reach $\$ 158$ billion in 2020 (United States Department of Health and Human Services, 2011). Using the demographic data form U.S. population, medical expenditures for cancer in the year 2020 could be very costly due principally to the use of the newly developed tools for cancer diagnosis, treatment and follow-up.

\section{WHY THE ESCALATING COST OF BREAST CANCER?}

The clinical diagnosis of breast cancer can be quite demoralizing to the patient. The exorbitant cost of cancer management has been reported to lead to imminent bankruptcy. Breast cancer economic burden for the United States exceeds \$209 billion a year in lost productivity and medical expenses. Even breast cancer patients with employer-sponsored insurance policies still pay excess of $\$ 6553$ out of pocket for treatment (Vann and Coping, 2011). Many of the drugs for the treatment of breast cancer such as tamoxifen, raloxifen, herceptin (trastuzumab) are all very expensive. The generic versions of these drugs hardly exist. Even patients who are eligible for Medicaid and Medicare pay more out of pocket expenses than patients who use employer-sponsored insurance. Group insurance on average for all patients covers about $80 \%$ of the cost. Whereas Medicare covers about $65 \%$ of the cost without Medicare part D and prescription drugs (Vann and Coping, 2011). Use of Magnetic Resonance Imaging (MRI) to enhance the precision of diagnosis is extremely expensive for most patients either in the United States or from any developing nation. Although Federal Law now requires insurance to cover the cost of reconstructive surgery, for both breasts, incidental expanses now compel most women to seek treatment therapy from faith and other traditional healers. To say the least, many indigent women now avoid use of prescription drugs and do not comply with visiting their oncologists. Many families currently face the dire choices of cancer treatment and bankruptcy and foreclosures.

\section{PRIMARY PREVENTION}

As the nation confronts massive budgetary deficits; the contributors to these financial constraints include the exorbitant cost of health care and defense budget. It seems logical that primary prevention of cancer and other chronic and degenerative diseases can significantly reduce the financial resources expended on health care services if preventive approaches are accorded priority.

It is most prudent for an individual to become cognizant of health risk associated with different work-related functions and choices of specific allures. Such health risks could include use of tobacco, excessive use of alcohol, eating habits, food items with high cholesterol content and lipids. Particularly, out-of-patient care is a tremendous cost saving measure because of the inflated cost of hospital beds, exposure to unnecessary superfluous medical technologies and the inadvertent risk of nosocomial and iatrogenic diseases which increase national medical expenses exponentially. Through self-examination and health education, one is able to avoid the emergency room services for life-threatening diseases. Besides, such emergency services are two to three times more costly than services provided in the comfort of a visit to one's family physician. The other contributory factors to the escalating cost of cancer treatment include 1)-the delay in detecting the incipient stage of onset of breast cancer, 2) use of more expensive diagnosis and the treatment by using very expensive cytotoxic endocrine drugs and invasive surgery which augment the cost of treatment. The incorporation of 30 minutes of exercise daily can boost our immune system. 
Regular visits to physicians particularly, the oncologists to examine and pinpoint the onset of incipient stage of breast cancer can pre-empt such abnormality before it progresses toward the critical stage in the natural history of breast cancer. Proficient knowledge about breast self-examination can protect any potential patient and alleviate associated anxiety among the at risk group. Scientific studies have revealed that upto $50 \%$ of cancers can be prevented if one complies with daily exercise, consumption of diet rich in phyto-nutrients and abstention from tobacco. The use of family history techniques can quicken early detection and regular breast self-examination can lead to early detection and efficient diagnostic intervention by an oncologist.

\section{BREAST CANCER CHEMO-PREVENTION}

Chemoprevention is the medical intervention which reduces breast cancer risk for women at high risk of developing the disease. The medications routinely prescribed to patients include Tamoxifen and raloxifene Evista. Tamoxifen blocks the growth and progression of many breast tumor. Raloxifene (Serms) is another drug used to assist patients to prevent the onset of breast cancer. These drugs must be taken as one pill per day for five years for optimum effectiveness. Eligible patients for Tamoxifen are at risk patients who have the risk score, greater than $1.66 \%$ using the Gail Model. As shown in table 1, the Gail Model is a tool that clinically trained physicians can use to predict the potential risk of developing breast cancer based on key variables such as age, reproductive history, parity and family history. Other ancillary risks include a breast biopsy which reveals precancerous conditions, such as atypical ductal hyperplasia or tubular carcinoma in-situ (LCIS) (Mayo Clinic, 2011).

\section{PERSONALIZED MEDICAL CARE OF PATIENTS WITH BREAST CANCER}

Pharmacogenomics is the science that investigates how individuals react to medication. Although the recent sequencing revealed the $99.9 \%$ semblance in human DNA make-up, the $0.1 \%$ single nucleotide polymorphism is about 1.4 million cells. Herein lies, the existing variability in the way humans react to medication, inherited allergies and environmental toxins. For example, an identical cancer drug such as herceptin (trastuzumab) may create distinct and variable side effects in different patients. One patient may experience lifethreatening side effects while the other could just exhibit mild side effects. Some cancer medications could shrink a tumor in one patient whereas in another patient, the same drug might be without any therapeutic benefit. These vagaries in medical treatment have increased patients' non-compliance with chemotherapy. Some become so frustrated and they are compelled to seek treatment from traditional healers, herbalists or faith healers. As a result of sequencing and pharmacogenomics, studies have revealed distinctive subtypes and shed light on cancer metastatic potential. Expression signatures are now used in the clinic to characterize and predict which breast cancer patients stand the chance of benefiting from adjuvant chemotherapy or surgery. In spite of the usefulness of the genome-wideassociation study, pharmacopgenomics is extremely most productive in enabling the oncologist to make best clinical judgment in successfully treating their breast cancer patients (Heger, 2011).

In "individualized medicine", pharmacogenomics presents the potential to identify medications which are tailored to the specific of needs of patients, based on their genetic profiles. There are professional caveats to establish a patient's comprehensive genetic profiles, there are multiple measurements required, they include: family history, parity status, socio-demographic characteristics, anthropometric data, molecular gene tests, assessment 
of biomarkers, sequencing of individual's genome and routine blood tests, and the packed cell volume among others. Dr. Elias Zerhouni, the former director of the National Institutes of Health stated in his "new strategic vision for medicine that the shift from a late curative paradigm to an early preemptive one is becoming increasingly possible" (Zerhouni, 2009). This is one mechanism of containing the escalating cost of cancer treatment nationwide. Besides, electronic health record is another technology to identify early onset and pre-empt the trajectory of cancer pathology and the ominous consequences of metastasis.

\section{ELECTRONIC HEALTH RECORD [EHR] TO REDUCE THE ESCALATING COST OF UNITED STATES HEALTH CARE}

The use of electronic health record would have been an unachievable task but for the visionary leadership of Presidents George Bush and Barrack Obama. However, the daunting challenges involve creating an acceptable definition of electronic health record, rectifying the problems of interoperability, developing efficient software that is user-friendly for gathering epidemiological data on family history and teaching clinicians an in-depth Medelian genetics to be able to interpret the cost of intra-familial risks associated with BRAC1/BRCA2 mutations and when to send out health alert reminder post cards to at risk breast cancer patients. To reduce the exorbitant cost associated with the management of BRCA1/2, there is the need for Information Technology (IT) personnel, physicians, nurses and hospital epidemiologists to collectively select the most user-friendly, and most succinct and costefficient software to be used in collecting family history data, and analyzing the comprehensive data compiled from at risk breast cancer patients.

To create clarity about this innovative technology to revolutionize our health care system, Electronic health record (EHR) has been defined by many scientists, adopting different perspectives. The Loeuring group defined electronic health record as "a repository of electronically maintained information about individual's lifetime health status and health care, stored such that it can serve the multiple legitimate users of the record" (Zerhouni, 2009). An EHR also implies an individual patient's medical record in digital format. EHR is a subset of Health Information Technology (HIT) which illustrates how any computer-based electronic gadget enhances the delivery of health care services. In his joint address to US congress in 2009, President Barack Obama stated that: "Our recovery plan will invest in EHR and other new technology which will reduce the medical errors and bring down the spiraling cost of healthcare and ensure the privacy of patient records and save lives" (Thomas-Brogan, 2009).

The 2009 economic stimulus package (HITECH act) passed by the US congress proposed providing incentives to physicians to adopt EHR. On the one hand, the act created financial incentives to physicians who adopt and use "certified EHRs." On the other hand, the intent was to reduce Medicare payment to erring physicians who will not comply with using EHR. To ensure that physicians receive EHR stimulus money, the HITECH act (ARRA) demands that physicians provide evidence of meaningful use of an EHR (Obama and Biden, 2009; Bush, 2011; Brailer, 2009).

Before the election of Mr. Barack Obama, as president of United States, President Bush had proposed several resolutions to enable both private and public sectors to make smooth transition to utilizing electronic medical record. He called for the completion and adoption of standards that will follow medical information to be stored and shared electronically while assuring privacy and security (Obama and Biden, 2009; Brailer, 2009). 
${ }^{*} \mathrm{He}$ requested that Health and Human Services (HHS) to negotiate and license a comprehensive medical vocabulary and make it available to the public at no cost. Owing to his intervention and leadership three other resolutions were made:

1. A patient's X-Ray can be sent electronically from one laboratory or hospital and can be read by patient's physician in his office.

2. Electronically, laboratory results can be sent electronically to the physician for immediate analysis, diagnosis and treatment and can be entered into a patient's EHR if one exists.

3. Prescription can be sent electronically to patient's pharmacist. This process expunges illegible handwriting of many physicians (Bush, 2004).

The two leaders have demonstrated their commitment to implementing the EHR because they seem to be aware of the multiple advantages of this communication technology. They are also aware of the capacity of EHR in allowing multiple uses to operate independently. They can simultaneously have access to health record in hitherto rural and medically isolated communities. There is easy retrieval of patient's medical record and updating of patients records. The other ancillary benefits of EHR include access to various decisionsupport tools and reduction in medical errors due to illegibility of physician handwriting. The major cost saving advantages is workforce reduction, due to the elimination of medical clerks, analysts, abstracters who are usually involved in cumbersome paper work. Such personnel can be redeployed to other sections of the hospitals.

\section{REDUCTION OF ESCALATING HEALTH CARE COSTS}

The administration of Medicare and Medicaid programs for eligible consumers is currently an accounting nightmare. Medical imaging is one of the rapidly growing costs of health care. Medicare part B increased from $\$ 6.80$ billion in 2000 to $\$ 14.11$ billion in 2006 . By implementing the EHR, access to patients' images using EHR will automatically eliminate duplication of expensive imaging. The nation's health care system can save over $\$ 11$ billion annually if all our medical payment transactions were handled electronically via direct deposit. The other serendipitous benefits are fast access to medical literature and the proliferation of incessant improvement in healthcare efficacy and reduction of medical errors because of their access to decision support (Brailer, 2009).

Table 1. Existing software with the potential to reduce cost of BRCA1/2 management

\begin{tabular}{|c|c|}
\hline Early Identification 0 & Risk Associated with \\
\hline CDC Genomics Family Healthy ware & Most Likely \\
\hline Understandingrisk.cancer.gov & Most Likely \\
\hline CancerGene & Most precise \\
\hline BayesMendel software BRCAPRO & Most Likely \\
\hline HugesRiskApps & Most Likely \\
\hline Gail Model & Most Likely \\
\hline
\end{tabular}


After the accomplishment of human genome sequencing by April 2003, many innovative information technology and biomedical scientists developed many software predicting intrafamilial risk of breast cancer. In fact, the scientific forerunner was CDC in 2004, when the United States Surgeon General Dr. Richard H. Carmona advised the nation to adopt the week of Thanksgiving as "Family Health week" (The National Children Study Committee Meeting, Atlanta GA, 2004). The predictability of the CDC family history public health initiative is at best useful for those physicians who are proficient in Mendelian genetics.

The Bayes Mendel software is equally useful mostly when used by competent nurses and physicians to perform the precise analysis of actual risk (USPHS, 2011). Many researchers have argued that the Cancergene is probable the best accepted method for computing breast cancer probabilities (Drohan et al., 2009). The Hughes Risk Apps which has the capability to import data from many other packages such as My Family Health Portrait via an HL7 interface is equally as useful (Salow, 2007). The Gail model which provides many variables which are precursors for breast cancer has the potential to predict BRCA1/BRCA2 whereby the oncologist can apply such knowledge for both primary and chemoprevention (USPHS, 2011; Drohan et al., 2009).

However, many primary care physicians are not geneticists, neither are their clinical nurse assistants who are the first point of contact to the anxiety-ridden potential cancer patient. In the age of genomic medicine, the limitations of the primary care physicians and other medical doctors who are not clinical geneticists but are compelled to establish contact with cancer patients must be recognized. Epstein has provided cautionary insights into the treatment of patients who suffer from diseases associated with their inherited genes (Epstein, 2006). According to him, it is the "Clinical medical geneticists that are able to (a) diagnose and manage genetic disorders; $(b)$ provide patient and family counseling; $(c)$ use their knowledge of heterogeneity, variability, and natural history of genetic disorders in patient-care decision making; (d) elicit and interpret individual and family medical histories; (e) interpret clinical genetic and specialized laboratory testing information; $(f)$ explain the causes and natural history of genetic disorders and genetic risk assessment; and $(g)$ interact with other health-care professionals in the provision of services for patients with genetically influenced disorders" (Epstein, 2006).

Regarding the software in Table 1, physicians who have received the adequate training in these pertinent concepts in medical genetics can easily recommend primary preventive approaches and chemoprevention to contain the expensive cost of cancer treatment. Therefore, the training of the new generation of physicians in this genomic age, must take into cognizance, the inclusion of advanced genetics into the curriculum of medical schools. Such comprehensive genetic education should incorporate education in the basic sciences and clinical areas pertinent to medical genetics, including mendelian genetics, cytogenetics, diagnosis and treatment of inborn errors of metabolism, molecular diagnosis, syndrome identification and dysmorphology, teratology, reproductive genetics, congenital malformations, multifactorial disorders, mental retardation and developmental disabilities, genetic screening, social and ethical issues in medical genetics, genetic counseling, and quantitative human genetics" (Epstein, 2006).

\section{LIMITATIONS}

Existing disadvantages of EHR include the cost of initial establishment and the technical challenges of implementation of an electronic system. Physicians need extra time away from 
their clinical duties to learn the new technology. Vast majority of health care transactions in the U.S. still operate using the paper format with illegible physician prescriptions. This obsolete process has been in existence for well over 50 years. By 2004 when President George Bush laid the groundwork for the establishment of the electronic health record, \$10billion was earmarked annually for the project. But by 2009, President Barack Obama continued with the same initiative to computerize the nation's health care system in five years. The challenges had become very obvious as only $8 \%$ of the nation's 5000 hospitals and $17 \%$ of its 800,000 physicians currently use the version of common computerized record keeping system. Existing drawbacks that are quite problematic consist of the dearth of IT workers, and the engineers to develop and implement the system. Several independent studies conducted by the Harvard Rand Group and the Commonwealth Fund have revealed that Obama's proposal could cost at least $\$ 75$ billion to $\$ 100$ billion over ten years for hospitals to aggressively implement the plan. However, as a component of the $\$ 2$ trillion a year health industry, a full-fledge EHR system will necessitate spending over 100 billion (Brailer 2009).

\section{CONCLUSION}

The initial investment and the process for implementation of the EHR nationwide could be very expensive; researchers have predicted that the accomplishment of this project could save the nation upward of $\$ 77$ billion annually. The commitment of IT engineers, administrators, physicians and other health workforce will expunge the ills of waste, medical errors, contain cost and associated inefficiencies. The other mechanisms of reducing the spiraling cost of health care will necessitate the adoption of public health interventions to eliminate preventable causes of death and disabilities such as heart diseases, cancers, and other chronic and degenerative diseases. The focus on primary preventive strategies has not been comprehensive enough.

Through the use of social engineering, mostly elderly can be provided specific incentives to exercise on a regular basis, engage in yearly check-up and breast-self examination. Young women and men of child-bearing age must be educated about the need to participate in breast cancer primary preventive education. Physicians and other health personnel must collaborate in understanding the role of pharmacogenomics in personalized and participatory medicine so as to maximize health benefits and minimize harm to the public.

\section{REFERENCES}

American Cancer Society Inc. Cancer Statistics. (2009). A presentation from the American Cancer Society. American Cancer Society Inc., 1-20.

Antoniou, A., Sinilnikova, O., McGuffog, L., Healey, S., Nevanlinna, H., Heikkinen, T., Simard, J., Spurdle, A. Beesley, J., Chen, X. (2009). Common variants in LSPI 2q35 and 8p24 and breast cancer risk for BRCA1 and BRCA2 mutation carriers Human Molecular Genetics 18, 22 doi: 10.1093/hmg/ddp372.

Brailer, D. (2009). Complete the work on health information technology Health affairs, The policy journal of the health sphere http://health affairs.org/blog/2009/01/14/completethe- work -on- health-information-technology.

Bush, G. (2004). Transforming health care: The president's health information technology plan. http:// www.whitehouse.gov/infocus/technology/economic policy200404/chip. 
Drohan, B., Ozanne, E., Hughs, K. (2009). Electronic Health Records and the management of women at high risks of hereditary breast and ovarian cancer. The Breast Journal, 15 suppl. 1, 46-55.

Ebomoyi, E. (2005). Genomics application in public health across all population; environment and work settings; Continuing education institute ; presented at the American public Health association 133rd annual Meeting and Exposition, Philadelphia, PA; www.apha. Confex.com'apha/133am/tech program/sessions_16480html_6k.

Epstein, C. (2006). Medical genetics in the genomic medicine of the 21st century. Am. J. Hum. Genet, 79, 1-8.

Gerencher, K. (2009). Many aren't taking necessary steps to avoid preventable cancer deaths. The San Diego Union-Tribune. http://www.signonsandiego.com/uniontrib20060425/news/news_1c25cancer.html

Gilpin C, Carson N, Hunter A. (2000). A preliminary validation of a family history assessment form to select women at risk for breast or ovarian cancer for referral to a genetic center. Clin Genetic,58,299-308.

Heger, M. (2011). Duke team says sequencing may be better than GWAS at pinpointing diseases-causing variants. GenomeWeb. Http://www.genomeweb.com/print/965719Retrieved.

Malone, K., Daling, J., Doody, D., Hsu, L., Bernstein, L., Coates, R., Marchbanks, P., Simon, M., McDonald, J., Norman, S., Strom, B., Burkman, R., Ursin, G., Deapen, D., Weiss, L., Folger, S., Madeoy, J., Friedrichsen, D., Suter, N., Humphrey, M., Spirtas, R., Ostrander, E. (2006). Prevalence and predictors of BRCA1 and BRCA2 mutations in a population based study of breast cancer in White and Black American women ages 35 to 64 years. Cancer Res., 16, 8297-3088.

Mariotto, A. (2011). Cancer care costs could exceed \$200Billion by 2020 Health Imaging and Information Technology http://www.healthimaging.com/index.php?option=com_article\&article.

Massachusetts Hospital Association Public hearing on Health Information Technology for economic and clinical health Act $(\mathrm{HITECH})$ provisions of the American recovery and reinvestment Act of 2009(ARRA) New England Executive Park, Burlington MA 018035096 www.mhalink.org Pp1-3.

Mausner, J., Bahn, A. (1987). Epidemiology: an Introductory Text. Philadelphia: W. B Saunders, 1-3, 3-35.

Mayo Clinic Breast. (2011). Cancer chemoprevention: Medicines that reduce breast cancer risk. http://health.msn.com/health-topics/center/breast-cancer-chemopreventionmedicine.

Mikail, C. (2008). Public Health Genomics: The Essentials. San Francisco, Jossey-Bass A Wiley Imprint, 8-10, 161.

National Cancer Institute Fact sheet Genetic testing for BRCA1 and BRCA2: It is your choice National Cancer Institute (NCl) Cancer information service. (2002). 1-10.

National Cancer Institute, United States, National Institutes of Health national cancer care expenditures were an estimated $\$ 104.1$ billion in 2006 . (2006). http://progressreport.cancer.gov/doc_detail.asp?pid

Obama, B., Biden, J. (2009). Barack Obama and Joe Bidden's plan to lower health care costs and ensure affordable, accessible health care coverage for and ensure affordable, accessible health care coverage for all. http:www.Barackobama.com.

Saslow, D., Boetes, C., Burke, W., Harms, S., Leach, M., Lehman, C., Morris, E., Pisano, E., Schnall, M., Sener, S., Smith, R., Warner, E., Yaffe, M., Andrews, K., Russell, C. (2007). American Cancer Society guidelines for breast cancer screening with MRI as an adjunct to mammography. CA Cancer J Clin, 57, 75-89. 
Thomas-Brogan, T. (2009). Health Information Technology Basics: a guide to principles and practice. Sudbury, Massachusetts: 82-89,135.

U.S. Preventive services Task Force (USPSTF) Recommendation statement Genetic risk assessment and BRCA mutation testing for breast and ovarian cancer susceptibility. (2005). http://www.ahrq.gov/clinic/uspstfo5/brcagenrs.htm. 1-18.

United States Department of Health and Human Services Biography of Vice admiral Richard H.Carmona, MD United States surgeon General Commander, USPHS commissioned Corps. (2003). http://www.hhs.gov/about/bio/sg.html.

United States Department of Health and Human Services, National Institutes of Health MD, Cancer costs projected to reach at least \$158billion in 2020. (2011). http://www.nih.gov/news/health/jan2011/nci-12htm.

Vann, M., Marcellin, L. (2011). Coping with the cost of breast cancer http://www.everydayhealth.com

Zerhouni, E.A. Fiscal Year 2009 directors Budget request statement. A new strategic vision for medicine (2009). http:// www.nih.gov/about director/budget request FY 2009 director's senate budget request.

(C) 2011 Ebomoyi; This is an Open Access article distributed under the terms of the Creative Commons Attribution License (http://creativecommons.org/licenses/by/3.0), which permits unrestricted use, distribution, and reproduction in any medium, provided the original work is properly cited. 